\title{
Idronoxil combined with cisplatin rescues refractory immune response in nasopharyngeal carcinoma
}

\author{
Ngar-Woon Kam ${ }^{1}$, Desmond Tae-Yang Hung ${ }^{1}$, Man Kin Yim ${ }^{1}$, Ka Chun Wu ${ }^{1}$, Xin-Yuan \\ Guan $^{1}$, Olivier Laczka ${ }^{2}$, John Wilkinson ${ }^{2}$, Sai-Wah Tsao ${ }^{3}$, Victor Ho-Fun Lee ${ }^{1}$, and Dora \\ Kwong ${ }^{1}$ \\ ${ }^{1}$ University of Hong Kong \\ ${ }^{2}$ Noxopharm Limited \\ ${ }^{3}$ The University of Hong Kong
}

August 12, 2020

\begin{abstract}
Background. Nasopharyngeal carcinoma is a chemosensitive cancer characterized by prominent lymphocytic infiltration. However, recurrence remains a major challenge in treating this disease. Here, we examined the effects of idronoxil (IDX) on tumor-lymphocyte interactions. Methods The antitumor activity of cisplatin and/or IDX was tested in authentic EBV-positive NPC-derived cell lines (C666, C17 and NPC43). We analyzed 2D monolayer and 3D spheroids cocultures of NPC cell lines with immune cells to assess the infiltration, activation, phenotype and function of $\mathrm{T}$ cells toward human nasopharyngeal tumors in vitro. Flow cytometric and immunohistochemical analyses were carried out to evaluate infiltrating $\mathrm{T}$ cells in subcutaneous mice models. Results. Administration of low-dose of IDX (1-2 $\mu \mathrm{M})$ not only sensitizes cancer cells to chemotherapy via apoptosis, but also promotes $\mathrm{T}$ cell proliferation and cytotoxicity. More importantly, combination treatment of IDX and cisplatin in cancer cells can enhance the expansion, trafficking and priming of CD4+, CD8+ and double-positive (DP) T cells. Mechanistically, we showed that the increase in migratory DP T cells was largely mediated by the CXCL10/type I interferon (IFN) axis, where IDX precipitates an elevation in IFN $\alpha$ levels and leads to CXCL10 induction. Additionally, IDX enhanced T-cell infiltration towards tumor spheroids with acquisition of increased tissue homing and functional abilities (attenuated CD62L and PD1 expression). Conclusions. Our findings underscore the potential of IDX to induce immunomodulatory responses, and in combination with conventional chemotherapies, enhance antitumor immunity.
\end{abstract}

\section{Main text}

\section{Introduction}

Nasopharyngeal carcinoma (NPC) is endemic in Southern China (Chang \& Adami, 2006). Patients with NPC are uniformly treated with high-dose radiation therapy, with additional (concurrent chemoradiation) or adjunct chemotherapy for locally advanced diseases (Blanchard et al., 2015; Shield et al., 2017). Unfortunately, $30-50 \%$ of patients still relapse within 5 years despite this radical intensive treatment (Sun et al., 2014). The main characteristic of this cancer is the high association between Epstein-Barr virus (EBV) infection and NPC risk, especially type III. Moreover, a hallmark of undifferentiated NPC (the commonest histological type in endemic regions) is substantial infiltration of immune cells into the tumor microenvironment (TME).

Consumption of soy is associated with many health benefits, including a modest reduction in the risk of developing certain cancers (Trock, Hilakivi-Clarke \& Clarke, 2006; Yan \& Spitznagel, 2005). Soy products provide the main sources of soy phytochemicals, including soy isoflavones which comprise predominately genistein, daidzein and glycitein. Idronoxil (IDX, also known as phenoxodiol) is a synthetic derivative of genistein (Joannou, Kelly, Reeder, Waring \& Nelson, 1995). Preclinical studies have shown that IDX causes 
apoptosis in a wide range of cancer cell lines and is 5-20 times more effective than genistein. IDX is also active in murine cancer models which can sensitize certain types of cancer to chemotherapy (Alvero et al., 2008). The activity of IDX is likely related to its inhibition of enzymes including sphingosine kinase and PI3 kinase which are over-expressed in cancer cells and responsible for regulating cell pro-survival mechanisms (Porter, Fairlie, Laczka, Delebecque \& Wilkinson, 2020; Yao et al., 2012). Another target of IDX is a protein found on the outer membrane of all cancer cells known as Ecto-Nicotinamide adenine dinucleotide oxidase disulfide-thiol exchanger type 2 (ENOX2) (Morre, Chueh, Yagiz, Balicki, Kim \& Morre, 2007). The binding of IDX to respective receptors can induce cell cycle arrest and promote DNA damage in cancer cells (Aguero, Facchinetti, Sheleg \& Senderowicz, 2005).

Whilst clinically tested in the past, IDX's journey was discontinued as a result of its low bioavailability in humans when administered either intravenously or orally, though strategies to overcome this issue are currently being explored. Strikingly, IDX has previously been reported to inhibit the proliferation and migration of human endothelial cells, thereby inhibiting angiogenesis (Gamble et al., 2006). Moreover, IDX can induce a range of interferon (IFN)-stimulated genes in immortalised mouse embryonic fibroblasts (Yagiz, Wu, Kuntz, James Morre \& Morre, 2007). These findings illustrate that IDX may be targeting the apoptotic machinery itself. Indeed, one report described activated T cells (Herst, Davis, Neeson, Berridge \& Ritchie, 2009) as a target for IDX and Georgaki et al. have also classified IDX as an immunomodulator through its capacity to stimulate the innate arm of the immune system, specifically the CD56 ${ }^{+}$natural killer (NK) cell population (Georgaki et al., 2009). Despite the augmentation of NK cell activity and T cells proliferation being critical for the treatment of tumors, the exact mechanisms by which IDX controls the TME and cancer progression remain unclear.

Here we determined the $\mathrm{IC}_{50}$ of IDX on NPC cell lines and showed that it could further sensitize the cells to apoptosis and inhibit the proliferation and migration in conjunction with cisplatin (cis) treatment. Our results also demonstrated a purported ability of IDX to re-educate the TME and elicit anti-tumorigenic effects. In this capacity, IDX itself could induce T-cell migration with augmented cytotoxic functions and proliferative capacity. While IDX treatment did not show significant impact alone, combination of both IDX and cis targeting was synergistic in regulating $\mathrm{CD} 8^{+} \mathrm{T}$ cell expansion. We next developed spheroid cocultures of NPC cells and immune cells to study the infiltration, activation and functions of these cells towards human tumors. Increased immune cell infiltrates in IDX-treated spheroids were enriched for double-positive (DP) $\mathrm{CD} 4^{+} \mathrm{CD} 8^{+}$cells, mediated by the up-regulated expression of IFN $\alpha /$ CXCL10. Particularly, the infiltrated DP cells demonstrated downregulated expression of both lymphoid node homing and dysfunctional markers, CD62L and PD-1. Thus, IDX kills cancer cells both directly and also indirectly, by evoking antitumor immunity by modulating T-cell infiltration into and migration within tumors. Additionally, IDX may be a tool to gather further insights concerning TME and the poor response of NPC cells to conventional chemotherapy.

\section{Materials and methods}

Cells, tissues and reagents

Four NPC cell lines (C17, C666, NPC43-ve and NPC43+ve), kindly provided by Professor George Tsao were cultured with RPMI1640 medium (Invitrogen) supplemented with $10 \% \mathrm{FBS}, 1 \% \mathrm{P} / \mathrm{S}$ and $4 \mu \mathrm{M}$ Y27632 ROCK inhibitor (Enzo Life Sciences,. ALX-270-333). NPC tissue samples used in this study were obtained from patients admitted to Queen Mary Hospital, the University of Hong Kong. Tissues were fixed and subjected to histological examination. The study has been approved by the Institutional Review Board of The University of Hong Kong/Hospital Authority Hong Kong West Cluster (HKU/HA HKW IRB, UW16-428).

IDX (provided by Noxopharm Limited, Australia) was suspended in DMSO at $40 \mathrm{mg} / \mathrm{mL}$ (stock solution) and stored at $-80{ }^{\circ} \mathrm{C}$. Serial dilutions of the stock solution were freshly prepared in complete medium and kept for less than 4 weeks at $-20{ }^{\circ} \mathrm{C}$. In all cultures, the final concentration of DMSO in the medium was $[?] 0.1 \%$. 


\section{Healthy donors blood cells}

Peripheral blood mononuclear cells (PBMCs) were isolated from buffy coat collected from Red Cross. Blood samples were diluted 1:2 with PBS/2 mM EDTA, layered on a density gradient (Ficoll-Paque-Plus density gradient) and centrifuged at $2000 \mathrm{rpm}$ for $25 \mathrm{~min}$. Interface containing PBMCs were carefully removed and cells were washed twice with PBS/EDTA followed by resuspension in RPMI1640 supplemented with $10 \%$ heat-inactivated foetal bovine serum (FBS) and $1 \%$ Penicillin/Steptomycin or cryopreservation in FBS/DMSO for future analysis.

\section{Cell migration, apoptosis and proliferation}

Cell proliferation was performed by seeding NPC cells into 6-well plates at the density of 1,000 cells/well, culturing for 7 days in the presence or absence of different concentrations of IDX with/without cisplatin, fixing in $4 \%$ paraformaldehyde and staining with $1 \%$ crystal violet. For the migration assay, NPC cell lines were seeded on the upper chambers of 24-well Transwell inserts (BD Biosciences) and allowed to migrate with/without combined drugs in the bottom well. Migrated cells were fixed and stained as described above. For the apoptosis assay, the apoptotic cells were quantified (percentage) using an Annexin V-FITC/propidium iodide (PI) apoptosis detection kit (BD Biosciences). Cells were seeded at a density of 2 x $10^{5}$ cells per well in 6-well plates. After treatment, cells were harvested and Annexin-V-FITC/PI labeling was performed according to the manufacturer's instructions. The stained cells were analyzed on a NovoCyte Quanteon flow cytometer (Acea Biosciences).

\section{Coculture and multi-parametric flow cytometry}

Cryopreserved PBMCs were thawed and resuspended in RPMI overnight in the presence of $120 \mathrm{IU} / \mathrm{mL}$ (Peprotech) before coculture. C17 and NPC43+ve NPC cell lines were pre-treated with indicated doses of IDX and/or cis for $24 \mathrm{~h}$ before coculturing with PBMCs via tranwell ( $3 \mu \mathrm{m}$ pore-size, Corning) for 3 days. For surface staining, PBMCs were collected, washed and resuspended in FACS buffer (PBS, $0.5 \%$ BSA and $2 \mathrm{mM}$ EDTA). Cells were pretreated with Fc block reagents (1:200; Biolegend), then stained with a mix of surface fluorophore-conjugated anti-mouse antibodies listed in Supplementary Table 1. For intracellular Ki67 staining, PBMCs were stimulated with anti-CD3/CD28 antibodies (BD Biosciences, both at 1:1000 dilution) and cocultured with tumor cells for 5 days. Brefeldin A $(1 \mu \mathrm{g} / \mathrm{mL}$; Sigma-Aldrich $)$ was added during the last $5 \mathrm{~h}$ of culture. After coculture, cells were washed, surface stained $\left(4{ }^{\circ} \mathrm{C}\right.$ for $\left.30 \mathrm{~min}\right)$ and then fixed with Cytofix/Cytoperm, followed by intracellular Ki67 staining. After the staining, cells were washed again prior to processing on a NovoCyte Quanteon flow cytometer (Acea Biosciences). Data analysis was perfoemed using FlowJo software (FlowJo, LLC, USA). Nonviable cells were excluded from the analysis based on forward and side scatter profiles, and 7-AAD dead cell stain.

\section{CD107a and granzyme-B functional assay}

A flow cytometry-based analysis of T-cell function was designed to detect CD107a membrane expression and intracellular granzyme B expression. Briefly, PBMCs were stimulated at $37^{\circ} \mathrm{C}$ overnight at a concentration of $5 \times 10^{5}$ cells $/ \mathrm{mL}$ in the presence of $25 \mathrm{ng} / \mathrm{mL}$ phorbol myristate acetate (PMA) and $1 \mu \mathrm{g} / \mathrm{mL}$ ionomycin. On the day of coculture, cells were stained with CD107a or its IgG1 k isotype (BD Biosciences). Brefeldin A and GolgiStop were added one hour later. After $72 \mathrm{~h}$, cells were washed once with FACS buffer, then surface stained $\left(4{ }^{\circ} \mathrm{C}\right.$ for $\left.30 \mathrm{~min}\right)$, followed by intracellular staining with granzyme B antibodies as mentioned above.

\section{Cytotoxicity assay}

NPC cell lines were labeled with $5 \mu \mathrm{M}$ CFSE (Sigma, catalog 21888), seeded in a 96-well plate at $10^{5}$ cells/well, and T cells previously cocultured with drug-treated tumor cells (generated as above) were added at the indicated ratios and cultured for $72 \mathrm{~h}$. Cells were stained with Fixable Viability Dye eFluor 780 (BD Biosciences), and staining was evaluated by flow cytometry as described above. The percentage of specific lysis was then calculated using the following equation: $\%$ specific lysis $=100 \times$ x $\%$ specific cell death - $\%$ basal cell death)/100 - \% basal cell death. Specific cell death was determined from viability dye staining of CFSE-positive cells in $\mathrm{T}$ cell coculture wells and basal cell death from wells lacking cocultured $\mathrm{T}$ cells. 


\section{Spheroid formation}

For the generation of tumor spheroids in $3 \mathrm{D}, 200 \mu \mathrm{L} /$ well of cell suspension in culture medium was seeded at cell densities of 20,000 cells/well. NPC (C17/NPC43+ve) cells were dispensed into Nunclon Sphera 96 wells plate (Thermo Fisher Scientific). The Nunclon Sphera surface was designed to cause minimal cell attachment with minimal extracellular matrix protein binding to the plate surfaces. Plates were incubated at $37{ }^{\circ} \mathrm{C}$ and $5 \% \mathrm{CO}_{2}$ in RPMI supplemented with FBS and ROCK inhibitor. To determine the $\mathrm{IC}_{50}$ values, spheroids were stained with $0.1 \mathrm{uM}$ Invitrogen Cell Event (diluted by PBS + 5\% FBS) and $0.2 \mathrm{uM}$ Lysotracker in 100 uL Gibco Fluorobrite DMEM with $10 \%$ FBS in the presence of indicated doses of IDX and cis.

\section{Spheroid coculture and imaging}

For coculture with PBMCs, PBMCs (HLA-matched if possible) pre-stimulated with IL-2 were added 3 days after spheroid formation at the ratio of 10:1. On the day of coculture, PBMCs were stained with MERCK Red Stain PKH26 following manufacturer instructions, and spheroids were stained with $0.1 \mathrm{uM}$ Invitrogen Cell Event, followed by drug administration. Image acquisition was performed once a day for 3 days. For the blocking assay, PKH26-stained and CellEvent-stained spheroids were cocultured in the presence or absence of anti-CXCL10 neutralizing antibody $(10 \mu \mathrm{g} / \mathrm{mL}$; RandD systems; catalog MAB266). Z-stack analysis was done by transferring the individual spheroid to a flat-bottom clear-bottom 96-well plate which helped facilitate imaging. Images were obtained at $10 \mu \mathrm{m}$ increments on the GE InCell6000 instrument using a 10x objective with an aperture of 0.6-0.8 AU. Then using free software provided by ImageJ (made available by the National Institutes of Health $[\mathrm{NIH}]$ ), stacking of the 10 images was performed, followed by application of maximum intensity projection along the $\mathrm{Z}$ axis by the Fiji plug-in "Particle Analyzer". 3D pictures were generated using "Clear Volume".

\section{Spheroid-infiltrating cells flow cytometric staining}

For flow cytometry analyses, 8 wells/condition were seeded. OUT and IN compartments were isolated by first pooling the 8 cocultures wells in eppendorf tubes. Spheroids were gently resuspended and left to sediment to the bottom of the tubes. Supernatant cell suspension constituted the non-infiltrating immune cells $(=$ OUT $)$. These steps were repeated twice with PBS in order to separate the spheroids from the noninfiltrating immune cells. Spheroids were then trypsinized to obtain a single cell suspension $(=\mathrm{IN})$ further analyzed by flow cytometry.

\section{Cytokine and antibody arrays}

Human Inflammation Antibody Array C3 (RayBiotech) was used to detect cytokines and factors in conditioned media from cells under indicated conditions. Cells were plated in 6-well and treated with IDX, cis or combination of both. $72 \mathrm{~h}$ later, culture medium was centrifuged prior to assay on the human cytokine array according to the manufacturer's guidelines.

\section{Western blot}

Cells were lysed in RIPA buffer containing protease and phosphatase inhibitors on ice for 45 min and collected by centrifuging at $150,000 \mathrm{rpm}$ for $15 \mathrm{~min}$ at $4{ }^{\circ} \mathrm{C}$. Protein concentrations were measured by the bicinchoninic acid protein assay kit (Pierce). Equal amounts of protein were solubilized in laemmili buffer, boiled for $5 \mathrm{~min}$ and then separated by $10 \%$ SDS-PAGE before transferring them onto nitrocellulose membranes. Membranes were blocked and probed with specific primary antibodies (Supplementary Table 2), detected by using appropriate HRP-conjugated secondary antibodies and visualized by incubation with enhanced chemiluminescence reagent (ECL, GE Healthcare) and exposure to film.

\section{Quantitative real-time PCR}

Total RNA (500 ng) was extracted using RNeasy mini kit (Qiagen) according to the manufacturer's instructions. Total RNA (500 ng) was reversed transcribed with Primescript RT (Takara) and the cDNA served as template for amplification and quantitative real-time PCR, which was performed using SYBR Green Master 
Mix (TaKaRa) on a LightCycler480 Roche. Relative quantification was measured using the Comparative Ct (Threshold Cycle) and normalizing the Ct values to GAPDH. All samples were analyzed in duplicate and the mean of the 2 runs were reported.

\section{Immunohistochemistry}

Sections of $4 \mu \mathrm{m}$ thickness were cut from formalin-fixed paraffin-embedded tissue blocks. The slides were deparaffinized in xylene, rehydrated, followed by an antigen retrieval step by heating at $95{ }^{\circ} \mathrm{C}$ for 45 min in citrate buffer (pH6). Endogenous peroxidase was blocked with peroxidase blocking reagent (DAKO) followed by non-specific binding protein block (DAKO, X0909). Sections were then incubated with mouse anti-human ENOX2 (1:500, abcam ab210751) overnight at $4{ }^{\circ} \mathrm{C}$. Slides were then washed, and secondary staining was performed with DAKO REAL EnVision Detection System (K5007, DAKO) and visualized with diaminobenzidine (DAB) according to the kit's instructions.

\section{In vivo tumor experiments}

All mouse work was approved by the Committee on the Use of Live Animals in Teaching and Research (CULATR) of the University of Hong Kong (protocol 4592-18). Experiments were performed in 8-weeks-old nude mice, in which thymus-derived $\mathrm{T}$ cells were not developed, but functional extrathymic $\mathrm{T}$ cells differentiation pathways, and low levels of $\mathrm{T}$ cells were retained. Thirty nude mice per group were inoculated once subcutaneously with $5 \times 10^{6} \mathrm{NPC}$ cell lines + matrigel (1:1) using a 29-gauge needle under anaesthesia. Around day 10, when we expect the tumors to start growing, mice were injected with IDX $10 \mathrm{mg} / \mathrm{kg}, 20$ $\mathrm{mg} / \mathrm{kg}$, Cis $(5 \mathrm{mg} / \mathrm{kg})$ or combinations of IDX and cis intraperitoneally. Drugs were injected every day for up to three weeks. Tumor growth was monitored every 2 days at the indicated time. Tumors were harvested to prepare paraffin tissue sections for immunohistochemical staining. Spleen cells were mechanically disaggregated, whereas isolation of tumor-infiltrating leukocytes required both mechanical and enzymatic digestion by using $1.5 \mu \mathrm{g} / \mathrm{mL}$ collagenase IV (Roche), $0.8 \mathrm{mg} / \mathrm{mL}$ dispase (Invitrogen), and $0.1 \mathrm{mg} / \mathrm{mL}$ DNase I (Sigma). Tissues were incubated in digestion medium at $37{ }^{\circ} \mathrm{C}$ for $30 \mathrm{~min}$.

Single cell and microarray data collection

Single-cell data were obtained from GSE150430. Data from 15 NPC tumor and 1 normal biopsies were used for global analysis. Epithelial cells were identified using EPCAM, KRT5, KRT15 and KRT19. ENOX2 expression in malignant tumor cells were obtained from 11 patients. ENOX2 expression was indicated by color scale. Data visualizations were generated by plotly. NPC gene expression profiling datasets GSE34573, GSE53819 and GSE12452, and gene methylation profiling datasets GSE52068 were obtained from the gene expression omnibus (GEO, https://www.ncbi.nlm.nih.gov/geo/) database of The National Center for Biotechnology Information. GSE12452 contained 31 NPC specimens and 10 normal nasopharyngeal tissues, GSE53819 included 18 NPC and 18 normal specimens whereas GSE34573 included 13 NPC specimens and 3 normal nasopharyngeal tissues excised by laser microdissection. The datasets were based on the GPL570 [HGU133_Plus_2] Affymetrix Human Genome U133 Plus 2.0 Array platform. GSE52068 used Infinium Human Methylation 450K BeadChip and the probes targeted CpG sites of genes. CpG sites are usually found at or near transcription start site (TSS), so high level of methylation of CpG might lead to suppressed expression of RNA transcripts.

\section{Statistics}

Differences in quantitative variables were analyzed by the Mann-Whitney $\mathrm{U}$ test when comparing 2 groups and by the two-way ANOVA or Kruskal-Wallis with Dunn's post-test when comparing $>2$ groups when appropriate. A p value $<0.05$ was considered as statistically significant.

\section{Results}

IDX enhances the cytotoxicity of cisplatin against NPC cell lines 
The human parental NPC cell lines C17, C666 and EBV-positive/negative NPC cells (NPC43+ve/ NPC43ve) used in this study were all positive for ENOX2 expression (Supplementary Figure 1A and B). We first determine the drug concentrations that caused inhibition of $50 \%$ cell viability $\left(\mathrm{IC}_{50}\right)$. IDX inhibited proliferation of NPC43-ve, NPC43+ve, C17 and C666 cells over 120 hours with $\mathrm{IC}_{50}$ values ranging between 2-4 $\mu \mathrm{M}$ (Figure 1A); however, NPC43-ve showed higher resistance to IDX treatment. Based on this initial data, doses ranging from 1-4 $\mu \mathrm{M}$ were selected and used to further assess the antitumor effects on cell migration and apoptosis. Cancer cells treated with IDX showed significantly reduced migration compared with untreated cells, in a dose-dependent manner (Figure 1B). Likewise, IDX dose-dependently increased late-stage apoptotic cells compared with the untreated control. (Figure $1 \mathrm{C}$ and D). The cis demonstrated diverse cytotoxicity with $\mathrm{IC}_{50}$ values ranging from $0.2-1.2 \mu \mathrm{M}$ in the four tested cell lines (Figure $1 \mathrm{E}$ ). In contrast to IDX, NPC43-ve cells demonstrated pronounced sensitivity, suggesting that cis might be more effective in killing EBV-negative cells. A combination cytotoxicity study evaluated the interaction between IDX $(1,2$ and $4 \mu \mathrm{M})$ and cis $(0.6,1.2$ and $2.6 \mu \mathrm{M})$. A combination of IDX and cis enhanced the inhibition of cell proliferation (Figure $1 \mathrm{~F}$ ), with minimal impact observed when cells were co-treated with lowest dose of cis $(0.6 \mu \mathrm{M})$. Similarly, Figure $1 \mathrm{G}$ demonstrated that cell growth was not significantly impacted in cells treated with either cis at $0.6 \mu \mathrm{M}$ or IDX at $1 \mu \mathrm{M}$, while cis at $4 \mu \mathrm{M}$ exhibited far greater inhibition on cell growth. We therefore selected a combination treatment of cis $1.2 \mu \mathrm{M}$ with IDX at 2 or $4 \mu \mathrm{M}$ resulting in 10 -fold and 20-fold reductions in cell growth when compared to IDX and cis treatments alone, respectively (Figure $1 \mathrm{H})$. The combination treatment also resulted in a reduction in NPC43-ve and NPC43+ve cell migration by 6-fold (Supplementary Figure 1C).

\section{IDX-treated cancer cells induce $\mathrm{CD}^{+} \mathrm{T}$ cell activation concomitantly with enhanced cytotox- icity and degranulation}

Given that EBV infection is a critical event in NPC tumorigenesis, we then focused the subsequent functional assays on authentic NPC cell lines positive with EBV (C17 and NPC43+ve). To investigate whether IDX exerts an immunomodulatory effect that drives adaptive immune responses in NPC, we performed in vitro coculture of tumor cells with peripheral blood mononuclear cells (PBMCs) from healthy donors, with particular focus on T-cell functionality (Figure 2A). IDX modestly promoted CD4 ${ }^{+}$and $\mathrm{CD} 8^{+} \mathrm{T}$ cell proliferation in PBMCs alone, as evaluated by Ki67 labeling. This up-regulatory effect was significantly enhanced on $\mathrm{CD}^{+}$, but not $\mathrm{CD} 4^{+}$T-cells, when PBMCs were cocultured with IDX-pretreated cancer cells (Figure $2 \mathrm{~B})$. Surprisingly, the highest concentration of IDX $(4 \mu \mathrm{M})$ greatly impaired $\mathrm{CD} 8^{+} \mathrm{T}$-cell proliferation, suggesting that IDX doses [?] $\mu \mathrm{M}$ were toxic to T cells. This was consistent with Georgaki's finding where a high concentration of phenoxodiol was demonstrated to be toxic to PBMCs (Georgaki et al., 2009). To assess IDX's ability to induce cytolytic PBMC responses we examined granzyme B production in the T cells. Granzyme B was significantly increased in IDX-pretreated cancer cells at low-doses ( 1 and $2 \mu \mathrm{M})$, while a high dose of IDX markedly downregulated that expression, normalizing to baseline levels (Figure 2C). To confirm that we were measuring newly produced granzyme B, rather than residual unreleased granzyme B, within responding cells, we co-stained the cells with anti-CD107a to assess degranulation. The up-regulation of CD107a expression mirrored that of granzyme B (Figure 2D).

The CD8/CD4 T-cell ratio is increased by combination treatment of IDX and cis, potentially potentiating the tumor-killing ability of $T$ cells

On the basis of the above findings, we selected a cis concentration of $1.2 \mu \mathrm{M}$ (thereafter referred as Cis1.2) and IDX concentrations of 1 and $2 \mu \mathrm{M}$ (thereafter referred as IDX1 and IDX2) for the subsequent experiments. We first confirmed that the selected doses elicited $<5 \%$ toxicity toward immune cells (Supplementary Figure 2A). Next, we evaluated the effects of IDX, cis or IDX+cis combination treatments on immune lineage markers (CD4 T helper cells, CD8 T cytotoxic cells, CD14 monocytes, CD19 B cells and CD56 NK cells) within the cocultures. We first performed an unsupervised high-dimensional data analysis using the t-distributed stochastic linear embedding (t-SNE) algorithm (Figure 3A). cis1.2-treated cancer cells showed similar t-SNE coordination in clusters that overlapped with untreated cancer cells (DMSO). A unique pattern was identified when PBMCs were cocultured with IDX1-treated cancer cells, where both numbers of CD4 ${ }^{+}$and CD8 ${ }^{+}$ 
subsets were substantially diminished. This effect was restored when cells were treated with IDX2, which was further upregulated upon IDX+cis combined treatment (Figure 3B). We then focused on cells expressing CD4 and CD8. Figure 3C illustrates that treatment with IDX2 alone and IDX2+Cis1.2 significantly induced the frequency of $\mathrm{CD}^{+}$cells as compared to DMSO. Specifically, CD8/CD4 ratio in the cocultures increased upon treatment with IDX1 and IDX2+Cis1.2 (Figure 3D).

Having shown that treatment with IDX alone or IDX+cis in cancer cells could drive T cell induction, we next sought to determine whether the tumor-primed-T cells could elicit effector functions. PBMCs post-cocultured with treated cancer cells were collected and further expanded with IL-2 to achieve enriched primed-T cells. Based on the cytotoxicity assay, primed-T cells which were previously educated by IDX1/IDX2+cis1.2treated cancer cells were capable of killing tumor cells in an effector-to-target ratio-dependent manner (Figure $3 \mathrm{E})$. These data clearly indicate that IDX and cis treatment alone or in combination not only directly mediate tumor cell apoptosis, but can also prime T cells toward acquiring an increased killing ability, confirming the critical role of these treatments in making the tumor more susceptible to T-cell-mediated killing.

\section{IDX combined with cis endows $T$ cells with enhanced migratory capability via a CXCL10-type I IFN axis}

Tumor-infiltrating immune cells that can produce cytotoxic mediators are amongst the most critical players toward resolving tumorigenesis; therefore, we next investigated the migratory capacity of PBMCs in response to drug treatment. Substantial increases in the total number of migrating PBMCs towards IDX2+cis1.2treated cancer cells were observed compared to DMSO control (2.4-fold, $\mathrm{p}=0.0052$; Figure 4A). Similarly, an increased number of migratory $\mathrm{CD}^{+}$cells were observed in combination treatments (IDX1+cis1.2 and IDX2+cis1.2) $(53.03 \% \pm 8.859, \mathrm{p}=0.0236$ and $68.18 \% \pm 7.299, \mathrm{p}=0.0029$, respectively; Figure $4 \mathrm{~B})$. Comparing T cells subpopulations, exposure of IDX alone to tumor cells could dose-dependently enhance CD4 ${ }^{+}$ and $\mathrm{CD}^{+}$cell migration (Supplementary Figure 2B). Particularly, the increased proportion of migratory $\mathrm{CD}^{+} \mathrm{T}$ cell (mean $61.2 \% \pm 1.937$ and $64.99 \% \pm 8.539$, respectively) was slightly greater than $\mathrm{CD} 4^{+} \mathrm{T}$ cell (mean $36.66 \% \pm 9.446$ and $33.5 \% \pm 3.87$, respectively), but markedly lower than double-positive (DP) $\mathrm{CD} 4{ }^{+} \mathrm{CD} 8^{+} \mathrm{T}$ cell subsets (mean $78.39 \% \pm 11.81$ and $82.19 \% \pm 15.48$, respectively) upon combination treatments (IDX1+cis1.2 and IDX2+cis1.2) when compared to DMSO. These results suggest that combination treatment could favor the migration of DP T cells towards tumor site (Figure 4C-E, Supplementary Figure $2 \mathrm{C})$.

We next investigated the effect of IDX, cis alone or in combination on downstream signaling in cancer cells. The action of IDX has been suggested to be restricted to cells with defective DNA repair capacity, such as cancer cells and likely modulated by the stimulator of IFN genes (STING) signalling pathway. In Figure 4F, STING protein was constitutively expressed in NPC cell lines, with levels sustained with IDX1. STING expression was, unexpectedly, reduced upon IDX2 treatment, and to a greater extent, by cis1.2. We speculate that cis may compromise STING expression, impairing the type I IFN production pathway and suppressing proinflammatory chemokine production. As expected, administration of cis downregulated IRF3 phosphorylation at Ser396, but not Ser386, in NPC cell lines as compared to control and IDX treatment. This suppressive effect was also observed for the type I IFN protein IFN- $\alpha$, while its expression was restored upon combination treatment. Interestingly, the detection patterns of apoptotic caspase- 7 cascade were opposite to those of IFN- $\alpha$, where cleaved caspase- 7 was found to be greatly increased in NPC cell lines following cis treatment, as compared to control and IDX alone. This finding is consistent with those previously reported on the apoptotic caspase cascade functions where caspase- 7 was shown to potentially cleave and suppress STING-mediated type I IFN production (Ning et al., 2019; White et al., 2014). Notably, we observed that IDX2 alone greatly induced the production of IFN I downstream component CXCL10, known to be involved in $\mathrm{T}$ cells chemotactic recruitment into tumor (densitometries demonstrated a 2.93 -fold increase in protein expression as compared to DMSO; Supplementary Figure 2D) (Berghuis et al., 2011; Muthuswamy et al., 2012). We further demonstrated that CXCL10 was present in the supernatant of tumor cells, and that its upregulation was particularly influenced by IDX2 (1.5-fold) and IDX2+cis1.2 combination treatment (1.32-fold; Figure 4G) as compared to DMSO. Moreover, the levels of additional proinflammatory chemokines (CXCL8 
[1.93-fold and 2.11-fold, IDX2 and IDX2+cis respectively], CCL8 [1-fold and 1.17-fold], CXCL9 [1.26-fold and 1.18-fold], TNFSF1B [1.48-fold and 1.24-fold], and TNFRSF1A [3.66-fold and 3.45-fold]) involved in T cell activation and chemotaxis were found to be significantly augmented upon treatment with IDX, either alone or in combination with cis1.2.

\section{Increasing tumor cell apoptosis and tumor-infiltrating cells in nasopharyngeal cancer cell line- derived spheroids upon IDX treatment}

To mimic in vivo conditions, we examined the cytotoxicity of IDX and cis on NPC cells using threedimensional (3D) tumor cultures (Supplementary Figure 3A). We monitored the effect of increasing concentrations of IDX (Figure 5A, left panel) and cis (Figure 5A, right panel) on apoptosis using fluorescent probes (non-toxic CellEvent Caspase-3/7 Green and LysoTracker Deep Red) in combination with bright-field microscopy to perform persistent real-time spheroid imaging. The drug effect in the 3D "CellEvent," was used to build a dose-response curve to determine the $\mathrm{IC}_{50}$ values for cis at $1.4 \mu \mathrm{M}$ and IDX at $2.1 \mu \mathrm{M}$ (Figure $5 \mathrm{~B})$. Overall, the $\mathrm{IC}_{50}$ values were comparable between the spheroid-derived cells in $3 \mathrm{D}$ and $2 \mathrm{D}$ conditions.

We next explored the interactions between tumor spheroids and PBMCs through heterotypic cocultures. Spheroids generated from an NPC cell line cocultured with PBMCs obtained from healthy donors that were HLA-matched whenever possible. After coculture, we studied the cellular composition within infiltrated tumor spheroid by mechanically separating infiltrating cells (IN) and cells remaining in the medium (OUT) (Figure 5C). An increase in PBMCs infiltration in the tumor spheroids upon treatment was detected as early as 2 hours, while the number of infiltrated cells remained constant in the controls (DMSO) over 72 hours of coculture. We found that IDX alone and combination treatment significantly increased the influx of PBMC into the spheroids when compared to cis alone. Combination treatment resulted in elevated infiltrated

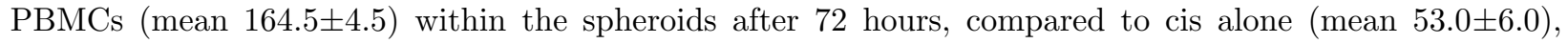
representing a significant $(\mathrm{p}=0.0149)$ 3.1-fold increase. Strikingly, PBMCs infiltrated in cis-treated tumor spheroids started to decline after 24 hours of incubation, where infiltrated PBMCs (mean 75.5土.5) within the control spheroids (DMSO) after 72 hours was higher when compared to cis alone. (1.4-fold increase; Figure 5D-E). Moreover, we also performed CellEvent staining on the coculture to confirm the induction of apoptosis and observed that spheroid infiltration was proportional to an active apoptosis process in tumor cells (Figure 5D, F). In agreement with our previous in vitro results (Figure 2D), the infiltrated PBMCs in IDX2+cis1.4-treated tumor spheroids showed increased levels, albeit modest, of CD107a compared to control (Supplementary Figure 3B).

We next hypothesized that IDX-conditioned tumor cells might activate PBMCs that in turn, infiltrate the tumor under the influence of chemokine gradients. Exposure of NPC cell lines to IDX resulting in the expression of the T-cell chemokines CXCL8, 9 and 10 supports this hypothesis (Figure 4G). To explore further, neutralizing antibodies against one of the chemoattractants, CXCL10, were added to the tumor spheroid cultures, which significantly reduced IDX-induced PBMC migration, and also, to a lesser extent, in the combination treatment, towards tumor spheroids. Collectively, these experiments refine our previous conclusions that IDX not only arrest tumor growth, but also induces the expression of T-cell chemoattractants, thereby enhancing T-cell infiltration, possibly augmenting further tumor killing.

\section{IDX administration induces differential expression of activation and homing markers in $\mathbf{T}$ cells infiltrates}

To assess which particular $\mathrm{T}$ cells infiltrate the spheroids upon IDX treatment, we determined $\mathrm{T}$ cell subsets based on expression patterns of 10 cell surface markers through flow cytometry. We observed dissimilar patterns of infiltrating cells following IDX treatment (blue) as compared to control (pink, Figure 6A). T cell phenotypes (CD3, CD4, CD8), followed by memory (CD45RO) and naïve (CD45RA) subsets were characterized which were thereafter gated for lymph node homing receptors (CD62L and CCR7) and activation markers (CD27 and PD1). Among those biomarkers, we observed substantial different surface expressions for CD3, CD62L and PD1 upon treatment with IDX in the cocultures. To technically validate our findings, we explored the $\mathrm{T}$ cell panelvia automatic gating by performing multivariate clustering of cells based on a 
self-organized map (SOM) algorithm. Representative rose-charts of the clusters illustrated an up-regulation of CD3 expression (Figure 6A, a), and reduction in CD62L expression (Figure 6A, b). Surprisingly, by performing k-means clustering and generating SOM, we observed fluctuated expression of PD1 in cocultured PBMCs upon IDX2 treatment. Interestingly, an inverse correlation was observed, such that upregulated PD1 expression correlated to reduced $\mathrm{CD} 45 \mathrm{RO}$ expression (Figure 6A, c).

By comparing $\mathrm{CD}^{+}$cell populations IN and OUT of the spheroids, we observed a significant increase in DP $\mathrm{T}$ cells in the tumor structure upon IDX2 treatment (Figure 6B). Additionally, tumor-infiltrating DP $\mathrm{T}$ cells displayed a pronounced reduction in CD62L, but not CCR7, expression compared to control (Figure 6C), suggesting that DP T cells could have a particular advantage toward spheroid infiltration following IDX treatment. Through gating analysis on $\mathrm{CD} 3 / \mathrm{CD} 4 / \mathrm{CD} 8^{+} \mathrm{T}$ cells following IDX-treated coculture, infiltrating $\mathrm{DP} \mathrm{T}$ cells showed decreased proportions of $\mathrm{CD} 45 \mathrm{RO}^{+} \mathrm{CD} 27^{+}$memory cells and increased proportions

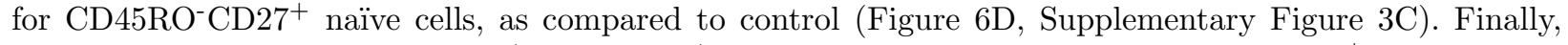
additionally to the above findings (Figure $6 \mathrm{~A}, \mathrm{c}$ ), we detected a significant reduction in $\mathrm{PD} 1^{+}$expression in total infiltrating memory cells compared to DMSO controls (mean 48.57 \pm 5.117 vs. mean $62.06 \pm 3.066 ; \mathrm{p}=$ 0.0086; Figure 6E). In contrast, we found significant up-regulation in $\mathrm{PD}^{+}$expression in naïve infiltrated cells upon IDX treatment as compared to DMSO (mean 62.10 \pm 7.049 vs. mean $43.57 \pm 3.870 ; \mathrm{p}=0.0017$ ). These results suggest that DP memory $\mathrm{T}$ cells are prone to infiltrate spheroids with a weak exhaustion profile upon IDX treatment.

\section{In vivo anti-tumor effect is associated with increased $\mathbf{T}$ cells infiltrations}

To evaluate possible antitumor effects of IDX on the growth of nasopharyngeal NPC43+ve tumors in nude mice, test animals were treated every-other-day with IDX and cis at a dosage of 10 and $20 \mathrm{mg} / \mathrm{kg}$ and $5 \mathrm{mg} / \mathrm{kg}$ weight/day, respectively. The growth curves demonstrated that high combination-treatment (IDX20+cis5) significantly decreased tumor growth, compared to the control group (Figure 7A, B). Although athymic nude mice are grossly deficient in peripheral $\mathrm{T}$ cells, the number of lymphocytes bearing $\mathrm{T}$-cell markers (CD4, CD8) increases steadily with age (Kennedy, Pierce \& Lake, 1992). Analogously, in our model, while $\mathrm{CD}_{4}{ }^{+}$(monocytes) and CD19+ (B cells) lymphocytes represented as the main two subsets, CD4 ${ }^{+}$and $\mathrm{CD}^{+} \mathrm{T}$ cells were also detectable in the circulation across all experimental groups. (Supplementary Figure 4A-C; before and after drug treatment for $\mathrm{CD}^{+} \mathrm{T}$ cells was $19.47 \pm 5.877$ and $18.01 \pm 5.411$, respectively; $\mathrm{CD}^{+} \mathrm{T}$ cells was $4.971 \pm 1.390$ and $9.675 \pm 5.213$, respectively). In relation to tumor-infiltrating lymphocytes (TILs), we observed significantly higher number of infiltrated $\mathrm{CD} 8^{+} \mathrm{T}$ cells within CD19- population in the tumor of IDX20+Cis-treated mice than that detected in the cis group (Figure 7C; $2.860 \pm 1.548$ and $8.482 \pm 1.758$, respectively). Importantly, the percentages of $\mathrm{CD}^{+} \mathrm{T}$ cells, albeit at low levels among all live TILs, was significantly higher in IDX20+cis-treated mice than that observed in the DMSO group (Figure $7 \mathrm{D} ; 1.668 \pm 0.5130$ and $5.400 \pm 1.426$, respectively), which was confirmed by immunostaining (Supplementary Figure 4D). However, there were no significant differences in the percentage of infiltrating $\mathrm{CD} 4^{+} \mathrm{T}$ cells among all groups.

Finally, we evaluated the clinical relevance of our signature findings by investigating the expression of ENOX2 as a potential link to IDX's pronounced anti-cancer effect in NPC. From a publicly available sequencing cohort GSE150430, we observed that malignant cells were the main cell type expressed ENOX2 at single-cell resolution (Figure 7E-F), this feature was further confirmed at protein level by histological staining (Figure 7G). Another three publicly microarray GEO datasets (GSE12452, GSE53819 and GSE34573) illustrated that ENOX2 gene was present at all stages of cancer progression (I-IV) and expressed at a significantly higher levels in tumors as compared to normal tissues, which was associated with decreased DNA methylation as evident in the GSE52068 dataset (Figure 7G). As such, the antitumor-responsive ENOX2 activity might be closely associated with NPC cancer, and thus IDX treatment could potentially elicit a tumor-killing and immune-mediated destruction in NPC tumor cells, while adding cis in combination could further enhance this effect.

\section{Discussion and Conclusions}


Previously, most studies with IDX focused on its anticancer properties in association with cell cycle arrest and via a range of apoptotic pathways in tumor cells, with little attention given to the drugs immunooncology potential. Therefore, for the first time, we have discovered that low-dose IDX acts as an immune stimulus that can induce immunogenic tumor cell death by facilitating T-cell priming and trafficking into tumors. Three experimental challenges were overcome to allow this finding: determining the appropriate concentration of IDX enabling an effective immune response in addition to its anti-tumor effect, elucidating the functions of IDX on tumor-lymphocyte interactions and finally, seeking the activation pathways involved in fighting NPC disease. The immune-stimulating properties of IDX might therefore lay a new foundation toward giving this unique molecule a second chance at contributing to the future of cancer treatment.

We first determined the cytotoxicity of IDX against a range of NPC cancer cell lines. Although IDX was found to be less cytotoxic than cis, the relative restricted range of $\mathrm{IC}_{50}$ values reflected its homogenous response across the various cancer cell lines. Additionally, we confirmed previous findings showing that IDX further sensitizes these cells to apoptosis in combination with a chemotherapeutic drug. Our interest in determining how IDX impacts immune function stems in part from results obtained during a past human clinical study. In 2004, phenoxodiol gained fast track approval from the FDA and was tested in phase II/III trials in humans (Mor, Fu \& Alvero, 2006) against various types of primary and metastatic cancers. However, lymphocytopenia was associated with phenoxodiol use (Herst, Davis, Neeson, Berridge \& Ritchie, 2009), suggesting that dosing was not fully optimised in these studies. Herein, we confirmed previous findings

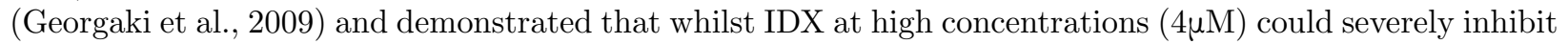
$\mathrm{T}$ cell proliferation in vitro, if given at appropriate dosages $(1-2 \mu \mathrm{M})$, IDX can act as an immune-enhancer in addition to its anticancer effects by allowing proliferating $T$ cells to unleash their cytotoxic ability. This finding further supports the application of this agent in future therapeutic interventions aiming to activate tumor-reactive lymphocytes.

As $\mathrm{T}$ cells are one of the main effectors of the antitumor immune response and are often suppressed during the disease, our finding that IDX positively modulates tumor-lymphocyte interactions is of significance. In our 2D coculture assay, we observed that conditioning of tumors with IDX, and to a larger extent upon combination treatment of IDX and cis, drives the expansion, activation and migration of T-cells. The effect is thought to be partially type I IFN/CXCL10-dependent in this study. To date, the STING field has been focused on the concept that it activates a predominant IRF3 response which has the potential to help recruit dendritic cells and $\mathrm{CD} 8^{+} \mathrm{T}$ cells to the TME, thereby promoting anti-tumor activities. While activation of STING in the myeloid compartment clearly promotes antitumor effects, the role of STING in tumor cells, and the role it may play in the antitumor response is starting to gain attention. Herein, we observed that low concentrations of IDX had no impact on endogenous STING expression, whilst higher IDX concentrations in combination with cisplatin down-regulated STING protein in NPC cell lines. Unexpectedly, a strong reduction in IFN $\alpha$ which is responsible for the upregulation of T-helper 1 cell chemokines, such as CXCL10, was only observed following cis treatment in NPC cells. Accordingly, the expressions of CXCL10, an important chemokine for the homing of antigen-presenting cells and trafficking of CD8 ${ }^{+} \mathrm{T}$-cells, were attenuated. This raised an important question as to why IDX is capable of sustaining IFN $\alpha$ and drive upregulation of CXCL10 expressions in NPC cells given that the STING pathway was partially impaired. As such, we drew our attention toward a recent discovery pointing at caspase cascade functions during apoptosis to prevent dying cells from producing IFN (White et al., 2014). Likewise, we observed that IDX modestly induced the release of mitochondrial DNA (mtDNA, data not shown), while cis was more likely to trigger cleaved apoptotic caspase- 7 , and the latter fed back to inhibit IFN $\alpha$ production by the dying cell. This finding highlights that, at least when it comes to the mode of action, IDX and cis exert their functions through different apoptotic downstream signaling pathways. Whether the elevation in IFN $\alpha$ caused by loss of the caspase cascade leading to the induction of chemoattractant is the result of upstream mitochondrial damage or an alternative signaling mechanism, and whether perturbation in STING activity might contribute to the immunological impact an apoptotic cell has on the NPC cells by inducing mtDNA remain to be established.

We further developed heterotypic cocultures of NPC tumor spheroids with T cells to study the infiltration, phenotype and function of infiltrates. Critically, this study showed that activated/memory DP CD4 ${ }^{+} \mathrm{CD} 8{ }^{+}$ 
T cells, acquiring lower expression of CD62L receptor and PD-1 marker, were able to infiltrate spheroids and kill tumor cells, and that CXCL10 was involved in these processes. Expression of CD45RO was the classical marker of primed memory CD8 $\mathrm{T}$ cells, which could be further divided into $\mathrm{CCR} 7{ }^{+} \mathrm{CD} 62 \mathrm{~L}^{+}$central memory and CCR7 $7^{-} \mathrm{CD} 62 \mathrm{~L}^{-}$effector memory cells based on the assumption that the presence of CD62L and CCR7 would facilitate entry of central memory cells to secondary lymphoid tissue from blood, whereas effector memory cells home to peripheral tissues. As such, our results pointed at another potential mechanism through which IDX could render the accumulation of exhausted PD1+TILs into tumor cells. Given the memory phenotype, cytotoxic profile, and migratory capacity that we observed for DP T lymphocytes, we could speculate that multiple immune activation circumstances exerted by IDX could favor the presence of these cells in the periphery of, and potentially inside, the TME. Further studies are deemed vital to better understand the mechanisms controlling the co-expression of CD4 and CD8 lymphocytes and whether such co-expression confers any functional advantage. We further confirmed our results in an in vivotherapeutic animal tumor model, confirming that this combination strategy was capable of inducing the accumulation of $\mathrm{T}$ cells within tumor sites and abrogate tumor growth.

Collectively, the multifaceted behavior of IDX provides the potential to synergize with, or complement, a wide range of cancer therapies, and overcome resistance mechanisms. It is therefore possible that IDX could condition or sensitize NPC patients to chemotherapy by converting "cold" or "altered" tumors into "hot" tumors, thereby increasing the chance of a durable response to targeted therapy.

\section{Bullet point summary}

\section{What is already known}

Myeloid-derived suppressor cells are responsible for

resistance to immunotherapy in patients with melanoma

Myeloid-derived suppressor cells are responsible for

resistance to immunotherapy in patients with melanoma

Myeloid-derived suppressor cells are responsible for

resistance to immunotherapy in patients with melanoma

- Nasopharyngeal carcinoma is an Epstein-Barr Virus-associated malignance characterized by prominent lymphocytic infiltration.

- Idronoxil (IDX) causes apoptosis in cancer cell lines and sensitise some cancers to chemotherapy/radiotherapy

\section{What this study adds}

- Combining IDX with cisplatin enhance T-cells expansion and accumulation within tumor and abrogate tumor growth

- The finding that IDX positively modulates tumor-lymphocyte interactions is of significance

\section{Clinical significance}

- Double action of IDX by converting "cold" micro-tumor into "hot", and leading to their destruction

- Immune-priming effect of IDX in combination with chemotherapy achieves major goal in immuneoncological drug development

\section{References:}

Aguero MF, Facchinetti MM, Sheleg Z, \& Senderowicz AM (2005). Phenoxodiol, a novel isoflavone, induces G1 arrest by specific loss in cyclin-dependent kinase 2 activity by p53-independent induction of 
p21WAF1/CIP1. Cancer Res 65:3364-3373.

Alvero AB, Kelly M, Rossi P, Leiser A, Brown D, Rutherford T, et al. (2008). Anti-tumor activity of phenoxodiol: from bench to clinic. Future Oncol 4: 475-482.

Berghuis D, Santos SJ, Baelde HJ, Taminiau AH, Egeler RM, Schilham MW, et al. (2011). Pro-inflammatory chemokine-chemokine receptor interactions within the Ewing sarcoma microenvironment determine CD8(+) T-lymphocyte infiltration and affect tumour progression. J Pathol 223:347-357.

Blanchard P, Lee A, Marguet S, Leclercq J, Ng WT, Ma J, et al. (2015). Chemotherapy and radiotherapy in nasopharyngeal carcinoma: an update of the MAC-NPC meta-analysis. Lancet Oncol 16: 645-655.

Chang ET, \& Adami HO (2006). The enigmatic epidemiology of nasopharyngeal carcinoma. Cancer Epidemiol Biomarkers Prev 15: 1765-1777.

Gamble JR, Xia P, Hahn CN, Drew JJ, Drogemuller CJ, Brown D, et al. (2006). Phenoxodiol, an experimental anticancer drug, shows potent antiangiogenic properties in addition to its antitumour effects. Int $\mathrm{J}$ Cancer 118:2412-2420.

Georgaki S, Skopeliti M, Tsiatas M, Nicolaou KA, Ioannou K, Husband A, et al. (2009). Phenoxodiol, an anticancer isoflavene, induces immunomodulatory effects in vitro and in vivo. J Cell Mol Med 13: 3929-3938.

Herst PM, Davis JE, Neeson P, Berridge MV, \& Ritchie DS (2009). The anti-cancer drug, phenoxodiol, kills primary myeloid and lymphoid leukemic blasts and rapidly proliferating T cells. Haematologica 94: 928-934.

Joannou GE, Kelly GE, Reeder AY, Waring M, \& Nelson C (1995). A urinary profile study of dietary phytoestrogens. The identification and mode of metabolism of new isoflavonoids. J Steroid Biochem Mol Biol 54: 167-184.

Kennedy JD, Pierce CW, \& Lake JP (1992). Extrathymic T cell maturation. Phenotypic analysis of T cell subsets in nude mice as a function of age. J Immunol 148:1620-1629.

Mor G, Fu HH, \& Alvero AB (2006). Phenoxodiol, a novel approach for the treatment of ovarian cancer. Curr Opin Investig Drugs 7: 542-548.

Morre DJ, Chueh PJ, Yagiz K, Balicki A, Kim C, \& Morre DM (2007). ECTO-NOX target for the anticancer isoflavene phenoxodiol. Oncol Res 16: 299-312.

Muthuswamy R, Berk E, Junecko BF, Zeh HJ, Zureikat AH, Normolle D, et al. (2012). NF-kappaB hyperactivation in tumor tissues allows tumor-selective reprogramming of the chemokine microenvironment to enhance the recruitment of cytolytic T effector cells. Cancer Res 72: 3735-3743.

Ning X, Wang Y, Jing M, Sha M, Lv M, Gao P, et al. (2019). Apoptotic Caspases Suppress Type I Interferon Production via the Cleavage of cGAS, MAVS, and IRF3. Mol Cell 74: 19-31 e17.

Porter K, Fairlie WD, Laczka O, Delebecque F, \& Wilkinson J (2020). Idronoxil as an Anticancer Agent: Activity and Mechanisms. Curr Cancer Drug Targets.

Shield KD, Ferlay J, Jemal A, Sankaranarayanan R, Chaturvedi AK, Bray F, et al. (2017). The global incidence of lip, oral cavity, and pharyngeal cancers by subsite in 2012. CA Cancer J Clin 67: 51-64.

Sun X, Su S, Chen C, Han F, Zhao C, Xiao W, et al. (2014). Long-term outcomes of intensity-modulated radiotherapy for 868 patients with nasopharyngeal carcinoma: an analysis of survival and treatment toxicities. Radiother Oncol 110:398-403.

Trock BJ, Hilakivi-Clarke L, \& Clarke R (2006). Meta-analysis of soy intake and breast cancer risk. J Natl Cancer Inst 98: 459-471.

White MJ, McArthur K, Metcalf D, Lane RM, Cambier JC, Herold MJ, et al. (2014). Apoptotic caspases suppress mtDNA-induced STING-mediated type I IFN production. Cell 159: 1549-1562. 
Yagiz K, Wu LY, Kuntz CP, James Morre D, \& Morre DM (2007). Mouse embryonic fibroblast cells from transgenic mice overexpressing tNOX exhibit an altered growth and drug response phenotype. J Cell Biochem 101: 295-306.

Yan L, \& Spitznagel EL (2005). Meta-analysis of soy food and risk of prostate cancer in men. Int J Cancer 117: $667-669$.

Yao C, Wu S, Li D, Ding H, Wang Z, Yang Y, et al. (2012). Co-administration phenoxodiol with doxorubicin synergistically inhibit the activity of sphingosine kinase-1 (SphK1), a potential oncogene of osteosarcoma, to suppress osteosarcoma cell growth both in vivo and in vitro. Mol Oncol 6: 392-404.

\section{Figure legends}

\section{Figure 1}

IDX enhances the anti-tumor effect of cisplatin in aggressive NPC cancer phenotypes in vitro

(A) Four NPC cell lines (NPC43 EBV-positive, NPC43+ve in blue; NPC43EBV-negative, NPC43-ve in red; C666 in black; C17 in green) were treated with increasing concentrations of IDX and cisplatin (cis) ranging from $1 \mu \mathrm{M}$ to $40 \mu \mathrm{M}$. The viability of cells treated with IDX for 5 days was analyzed by XTT cell proliferation assay. (B) Representative images of cell migration in $8 \mu \mathrm{m}$ transwell upon IDX treatment, stained with crystal violet, with the migratory cell counts shown next to the images. (C) Representative flow cytometric profiles showing Annexin V and PI staining of NPC cells following IDX treatment. (D) Bar graph represent the percentage of Annexin-V/PI positive NPC cells following IDX treatment. Asterisk to the right of bar indicate statistical difference compare to control DMSO in respective cell lines. (E) Viability of cells treated with cisplatin (Cis) for 5 days was analyzed by XTT assay. (F) Combinatorial treatment of IDX at 1,2, $4 \mu \mathrm{M}$ and Cis at 0.6, 1.2, 2.6 $\mu \mathrm{M}$ in four NPC cell lines by XTT assay. (G) Representative images of cell growth following IDX and/or cis treatment, stained with crystal violet. (H) Quantification of cell growth is shown. Results are representative of three independent experiments and presented as the means $\pm \mathrm{SD}{ }^{*} \mathrm{p}<0.05,{ }^{*} \mathrm{p}<0.01$.

Figure 2

IDX promotes $\mathrm{T}$ cells cytolytic potential following degranulation upon coculture with NPC tumor cells

(A) Flow cytometry plots of PBMCs alone and in coculture following IDX stimulation for 3 days. Gated frequencies represent the percentage of $\mathrm{CD} 4^{+}$or $\mathrm{CD} 8^{+} \mathrm{T}$ cells among Ki67, granzyme $\mathrm{B}$ and CD107a bright events. Bar chart of Ki67 (B), granzyme B (C) and CD107a (D) expression in CD8 ${ }^{+} \mathrm{T}$ corresponding to the above flow cytometry plots. Statistical significance was analyzed using 2 -way ANOVA, ${ }^{*} \mathrm{p}<0.05 ;{ }^{* *} \mathrm{p}$ $<0.005$ when compared with control (DMSO) in PBMCs cultured alone; \#p $<0.05$; \#\# p $<0.005$ when compared with control (DMSO) in cocultures.

\section{Figure 3}

\section{IDX combined with cis enhances $\mathbf{T}$ cells expansion with cytolytic potential in killing tumor cells}

(A) To capture and visualize all mononuclear cell subpopulations in a single two-dimensional (2D) map, representation of t-distributed Stochastic Neighbor Embedding (t-SNE) analysis of concatenated mononuclear cells (3,000 cells/sample) from PBMCs collected from cocultures was applied. T-SNE represent phenotypic markers defining specific cells and are assigned specific colors (CD19 B cells in purple; CD56 natural killer cells in pink; CD14 monocyte in green; CD4 T helper cells in orange; CD8 cytotoxic T cells in blue). (B) PBMCs cocultured with tumor cells following treatments as followed: IDX at $1 \mu \mathrm{M}$ and $2 \mu \mathrm{M}$ (IDX1 in orange and IDX2 in green) or cis at $1.2 \mu \mathrm{M}$ (cis1.2 in pink), combinatorial treatment with IDX1 $\mu \mathrm{M}$ and cis 
$1.2 \mu \mathrm{M}$ (IDX1+cis1.2 in purple) or IDX $2 \mu \mathrm{M}$ and cis $1.2 \mu \mathrm{M}$ (IDX2+cis1.2 in dark green), or left untreated with DMSO (blue). Differential expression patterns are shown. (C) Flow cytometry analysis of the frequencies of $\mathrm{CD}^{+}$and $\mathrm{CD} 8^{+}$cells in the cocultures. (D) CD8/CD4 ratio was calculated according to the cellular percentage changes between different treatments. (E) Representative flow cytometry plots of NPC cell line C17 (target cell; T) stained with CFSE before getting in contact with PBMCs (effector cell; E) collected from cocultures at indicated effector:target ratio (E:T) for 3 days. Cells were then collected and stained with fixable viability dye (FVD) and the dead cells (\%) were measured by flow cytometry. CFSE+ cells are target cells and FVD + cells are dead cells, such that dead target cells are represented by the CFSE + FVD +. (F) Quantitative data of \% lysis (in terms of CFSE+FVD+ cells) was obtained from three independent experiments. Statistical significance was analyzed using 2-way ANOVA, ${ }^{*}$ p $<0.05$; when compared with control (DMSO).

Figure 4

IDX combined with cis induces T cells migration towards tumor cells via CXCL10/type I IFN axis

(A) $\mathrm{CD} 45^{+}$for total leukocytes migrate towards C17 and NPC43+ve tumor cell lines following IDX and cis treatments alone or combined were measured in flow cytometry. (B) Specific migratory effects of the \% CD3 T cells/CD45 cells, ${ }^{* *} \mathrm{p}<0.01$ when compared to control (DMSO). (C) \% CD4 helper T cells/CD3 cells, (D) \% CD8 cytotoxic T cells/CD3 cells and (E) \% double positive CD4 CD8 cells/CD3 cells (E) relative control (DMSO) were quantified. * p[?]0.05 when compared to control (DMSO). (F) Tumor cells were pretreated with brefeldin $\mathrm{A}(5 \mu \mathrm{g} / \mathrm{mL})$ followed by treatment with the drugs alone/combined at the indicated concentrations for 8 hours Western blots were performed on the cell lysates with the indicated antibodies. p-IRF (396) and p-IRF (386) indicate phosphorylated serine 396 and 386, respectively. (G) Antibody array analysis of inflammatory cytokines in conditioned media from indicated NPC cell line following treatment with IDX, cis or combinations of both. Most prominently induced cytokines are highlighted in colored boxes. Quantitation of relative fold induction of cytokines compared with DMSO control cells were measured. Data are representative of two independent experiments. Statistical significance was analyzed using 2-way ANOVA ${ }^{*} \mathrm{p}[?] 0.05,{ }^{* *} \mathrm{p}<0.01,{ }^{* * *} \mathrm{p}<0.001,{ }^{* * * *} \mathrm{p}<0.0001$ relative to control.

Figure 5

High-content monitoring of drug effects in a 3D spheroid model upon IDX and cis treatments.

(A) Spheroids were pre-formed for 3 days with 20,000 cells/well in the presence of $0.1 \mathrm{uM}$ CellEvent and $0.2 \mathrm{uM}$ Lysotracker and then treated with indicated concentrations of IDX and cis. Representative images were acquired at day 3 using the InCell 6000. The intensity of C17 spheroid staining with CellEvent was proportional to IDX and cis concentrations. When LysoTracker was added to the spheroids, the staining pattern was complementary to that of CellEvent, with the LysoTracker constantly accumulating in the outer and presumably metabolically active layers of the spheroids. (B) CellEvent was used to determine the $\mathrm{IC}_{50}$ values for IDX and cis. (C) Schematic representation of the protocol used for the coculture of C17/NPC43+ve spheroids and PBMCs from healthy donors and subsequent imaging and flow cytometric analysis. (D) Representative images were acquired at the indicated time points using the InCell 6000 where PKH26stained and CellEvent-stained spheroids were cocultured. (E) Numbers of infiltrated cells were calculated by Fuji ImageJ software. (F) Apoptotic tumor cells were measured according to CellEvent intensity. (G) Pre-formed tumor spheroids were pre-stained with CellEvent followed by coculture with PKH26-stained PBMCs in the presence or absence of anti-CXCL10 $(10 \mu \mathrm{g} / \mathrm{mL})$ neutralizing antibody everyday throughout 3 days of coculture. Images were obtained at $10 \mu \mathrm{m}$ increments on the GE InCell6000 instrument using a 10x objective. Representative images of 10 stacked planes are shown. Representative images of 3D pictures were generated using "Clear Volume". (H) Quantification of immune cells infiltrating the spheroids upon IDX2 treatment alone or in combination of Cis1.4 was done. ${ }^{*} \mathrm{p}[?] 0.05$ when compared to IDX treatment alone.

\section{Figure 6}




\section{Activated/memory $\mathbf{T}$ cells are able to infiltrate tumor spheroids upon IDX treatment}

(A) To enable an unbiased analysis of data, we selected live cells and then used t-SNE to reduce dimensionality, followed by a graph-based heat-map clustering analysis. Representative t-SNE analysis of concatenated mononuclear cells $(2,000$ cells/sample) from "IN" compartment done by pooling 8 wells/condition of infiltrating tumor immune cells upon IDX treatment. Merged t-SNE plot (left panel) for drug treated and untreated cocultures, with each cluster indicated by a color (DMSO in pink; IDX2 in blue). Data were further clustered according to criteria used to define known T cell subsets: CD3, CD4, CD8, CD62L, CD27, CCR7, CD45RO, CD45RA and PD1. Automatic gating by Flowjo performed multivariate clustering of cells based on self-organized map algorithm and categorized all individual cells into 100 distinct clusters based on the surface expression marker proteins. Most prominent differential protein expressions CD3 a), CD62L b) and PD1 c) are highlighted in representative rose-charts boxes. (B) Flow cytometry analyses of T cells (respectively gated $\mathrm{CD}^{+}$) as well as $\mathrm{CD} 4^{+}, \mathrm{CD}^{+}$an double-positive $\mathrm{T}$ cells subsets (respectively gated $\mathrm{CD}^{+}{ }^{-} \mathrm{CD} 8^{-}, \mathrm{CD}^{-} \mathrm{CD} 8^{+}$and $\mathrm{CD} 4^{+} \mathrm{CD} 8^{+}$among $\mathrm{CD} 3^{+}$) percentages in the IN and OUT compartments, in the presence or without IDX at 72 hours. (C) Flow cytometry analyses of migratory T cells (respectively gated $\mathrm{CD}_{2} 2 \mathrm{~L}^{+} / \mathrm{CCR}^{+}$of $\mathrm{CD}^{+}$) on $\mathrm{CD}^{+}, \mathrm{CD} 8^{+}$an double-positive $\mathrm{T}$ cells subsets, percentages in the IN compartments, in the presence or without IDX at 72 hours. (D) Flow cytometry analyses of exhaustion $\left(\mathrm{PD}^{+}\right) \mathrm{T}$ cells phenotypes percentages (respectively gated $\mathrm{CD} 4^{+} \mathrm{CD} 8^{+}$of $\mathrm{CD} 3^{+}$) on $\mathrm{CD} 45 \mathrm{RO}^{+} \mathrm{CD} 27^{+}$ (memory $\mathrm{T}$ cells) and $\mathrm{CD}_{45 \mathrm{RO}^{-}} \mathrm{CD}_{2} 7^{+}$(naïve $\mathrm{T}$ cells ) subsets, in the IN compartments, in the presence or without IDX at 72 hours. (E) Quantitation of flow cytometric data on the percentage of $\mathrm{PD}^{+}$and $\mathrm{PD}^{-}$ memory $\mathrm{T}$ cells and naïve $\mathrm{T}$ cells in the presence of IDX relative to control (DMSO). ${ }^{*} \mathrm{p}[?] 0.05,{ }^{* *} \mathrm{p}<0.01$ when compared to DMSO control.

\section{Figure 7}

\section{Combinatorial treatment with IDX and cis inhibits tumor growth along with increasing T cells infiltration.}

(A) Representative mice and dissected tumors. For one mouse from the cis group, where the inoculated tumor was deeply located inside the organ tissue, that tumor could not be clearly dissected out and therefore was not its representative image is not shown. (B) Tumor volume during treatment. Tumor xenografts were established in athymic nude mice by inoculation of $\mathrm{C} 17$ cancer cells. After the tumors had grown to approximately $50 \mathrm{~mm} 3$, the animals were randomly divided into 6 groups ( $5 \mathrm{mice} /$ group), which were treated every-other-day with $5 \mathrm{mg} / \mathrm{kg}$ cis, $10 \mathrm{mg} / \mathrm{kg} \mathrm{IDX,} 20 \mathrm{mg} / \mathrm{kg}, 5 \mathrm{mg} / \mathrm{kg}$ cis plus $10 \mathrm{mg} / \mathrm{kg}$ IDX or $5 \mathrm{mg} / \mathrm{kg}$ cis plus $20 \mathrm{mg} / \mathrm{kg}$ IDX. Control mice received saline. Tumors were measured each other day over a total of 21 days after cancer cell inoculation and tumor volume was calculated. * p[?]0.05 and $* * * \mathrm{p}<0.0001$ when compared to control group. (C) Violin plot showing the percentage of infiltrating cells expressing CD14+ (monocyte) and CD19+ (B cells) in total live lymphocytes and cells expressing CD4 ${ }^{+}$, $\mathrm{CD} 8^{+}$and $\mathrm{CD}^{+} \mathrm{CD}^{+}$in $\mathrm{CD} 19^{-}$populations isolated from mice tumor, measured by flow cytometry. Each point represents one mouse in each group. ${ }^{* *} \mathrm{p}[?] 0.01$ when compared to cis group. (D) Flow cytometric analysis on tumor infiltrating CD8 $+\mathrm{T}$ cells upon aforementioned treatment in total live lymphocytes. * p[?]0.05 when compared to DMSO group. (E) Expression of ENOX2 at single-cell resolution from a publicly available sequencing cohort GSE150430. UMAP plot generated by using all cells harvested from 15 patients. Epithelial clusters enriched with mainly cells from tumor were circled and found to be the major cell type expressing ENOX2. (F) UMAP plot of malignant cells from 11 patients. Some patients including patient 11 and 13 were found to contain malignant cells with high expression of ENOX2. (G) Representative tumor histology staining of NPC clinical tissues for ENOX2 expression. (H) Analysis of ENOX2 gene expression and methylation from four independent public microarray datasets GSE34573, GSE53819, GSE12452 and GSE52068. * p[?]0.05 when compared to corresponding normal tissue. 


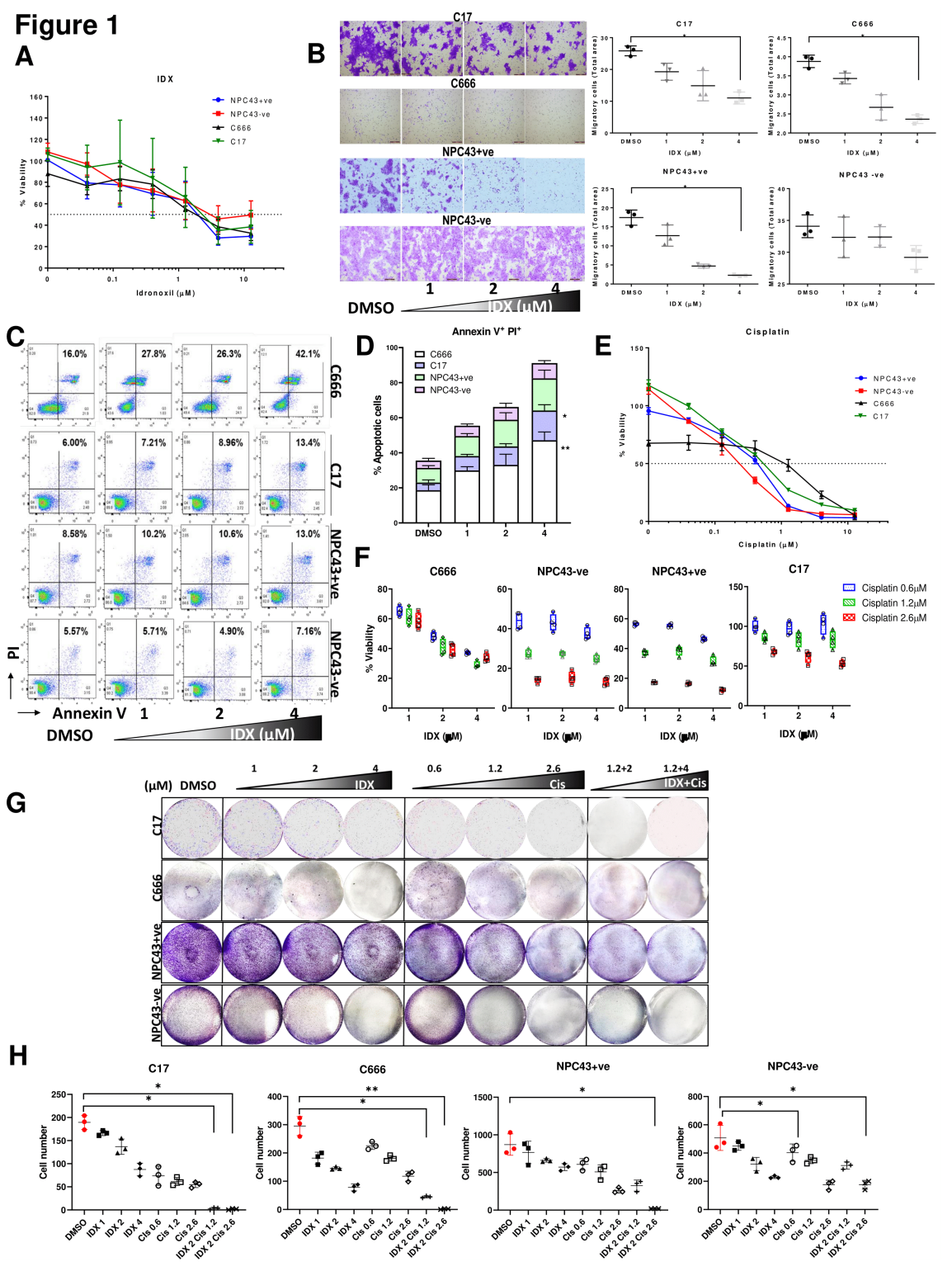


Figure 2

A
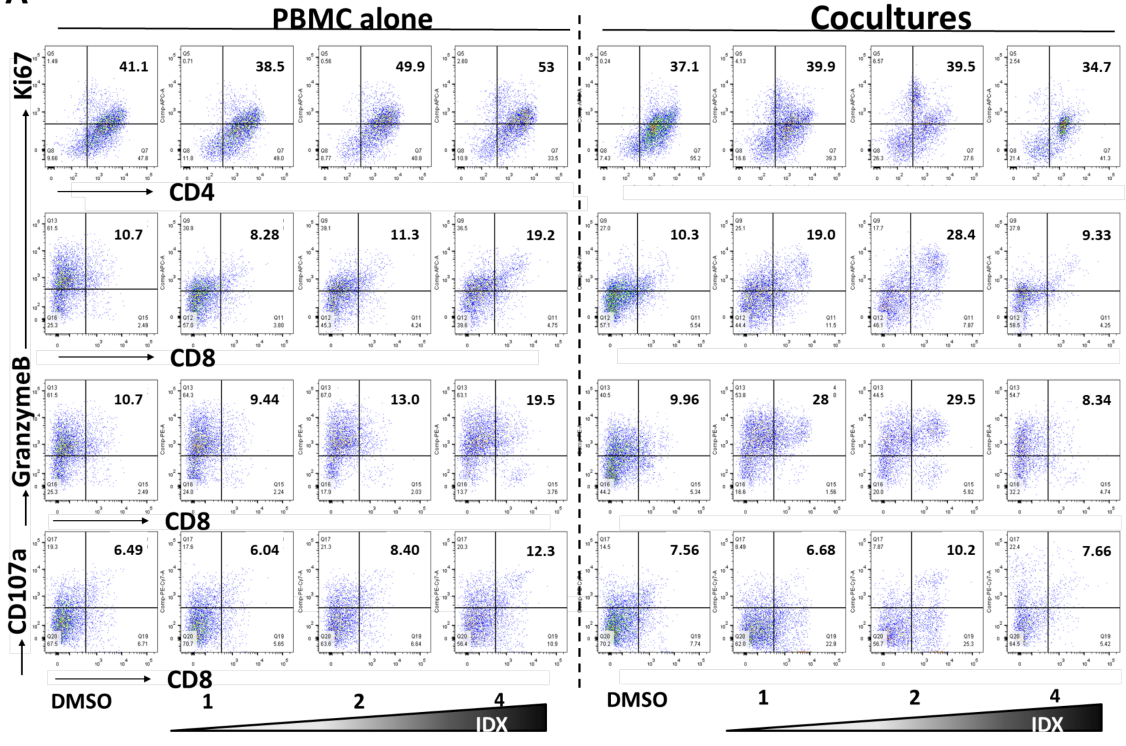

B

C
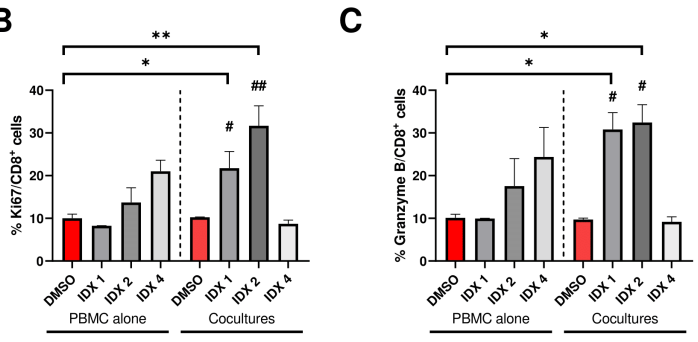

D

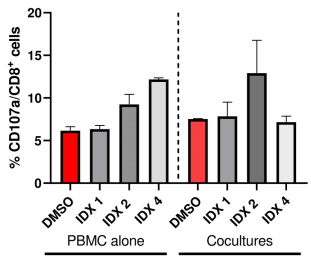


Figure 3
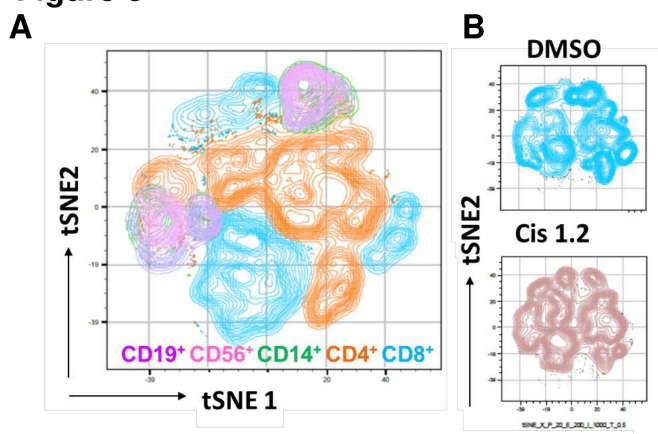

C
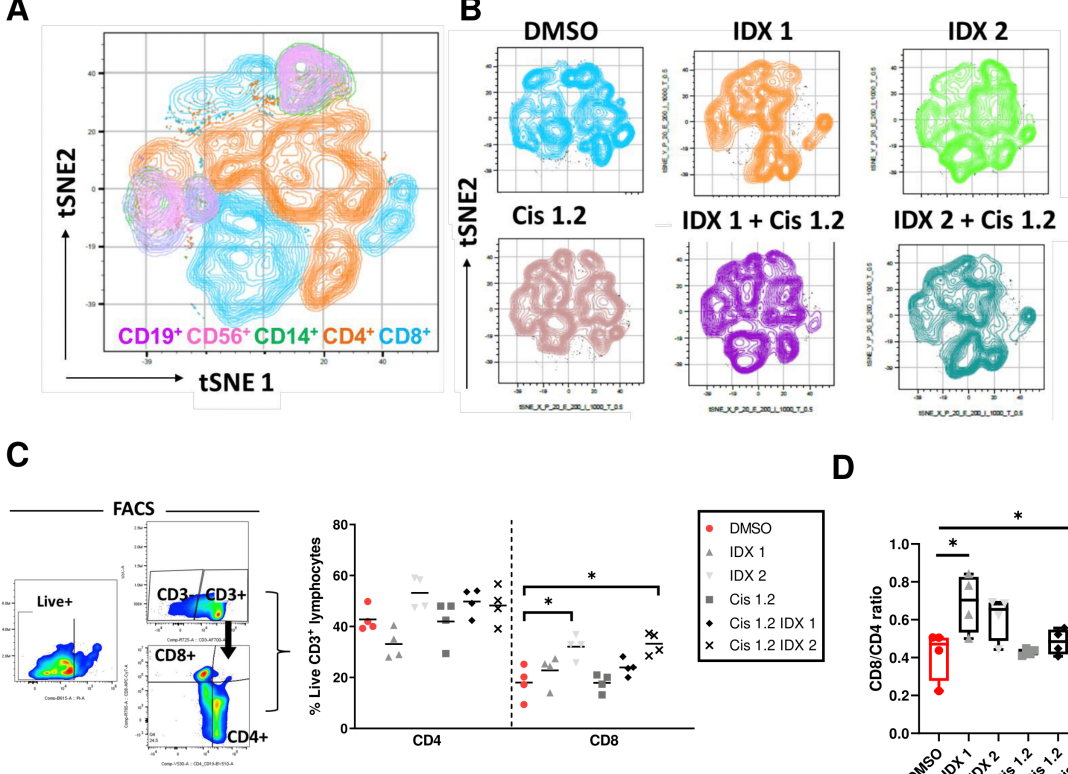

D
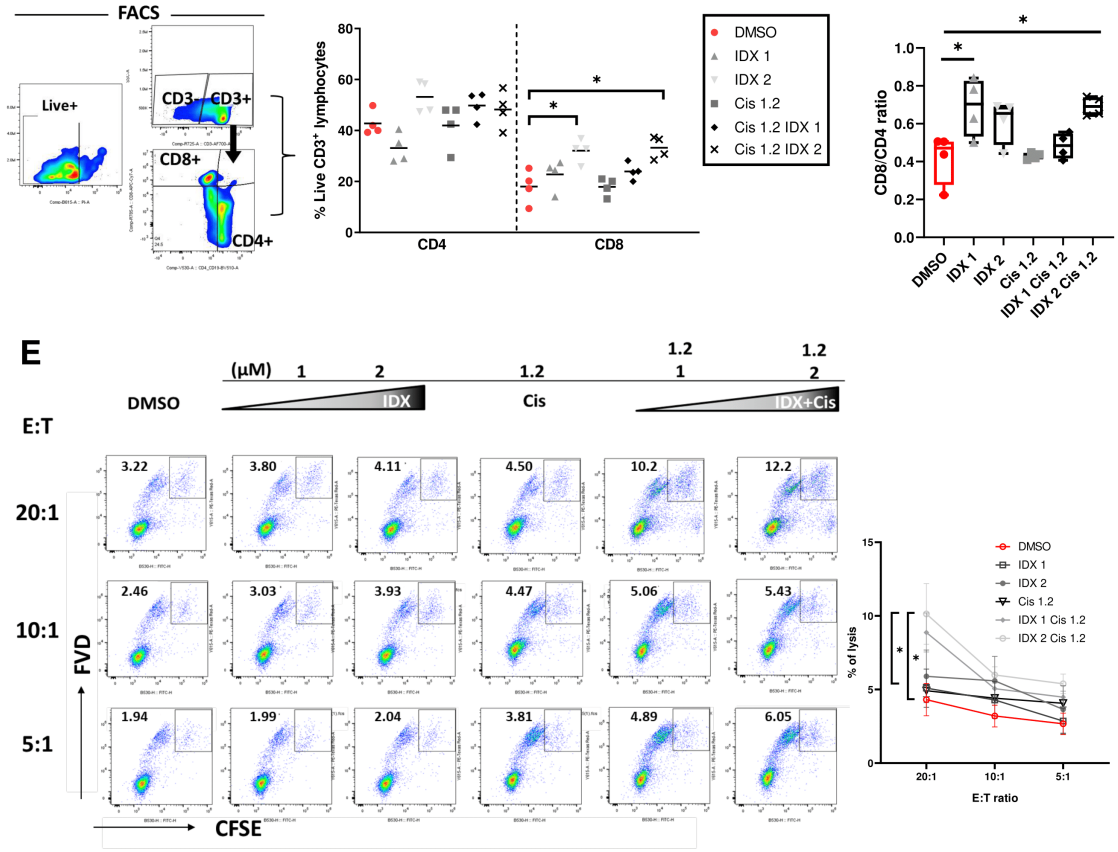
Figure 4
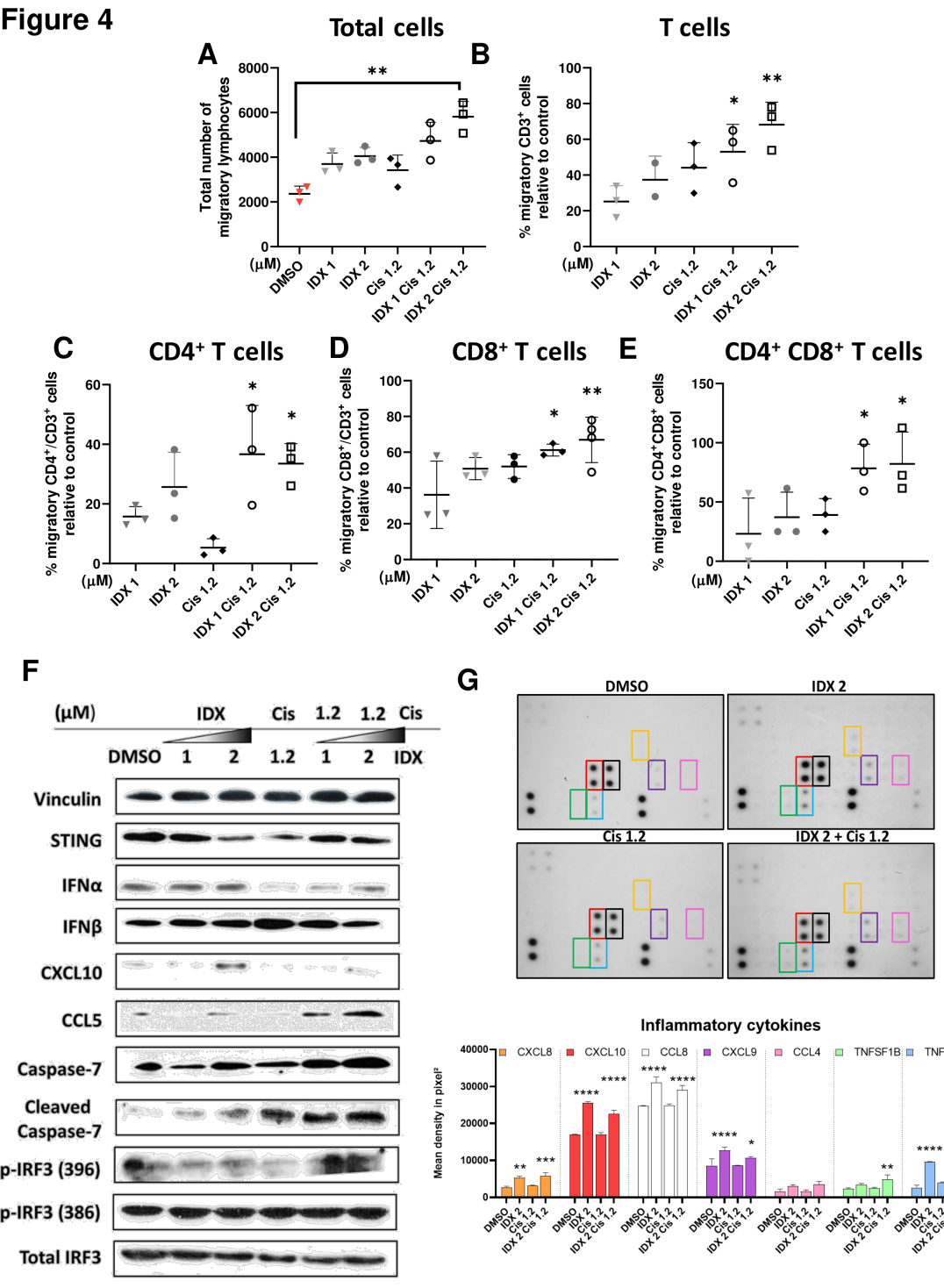

G
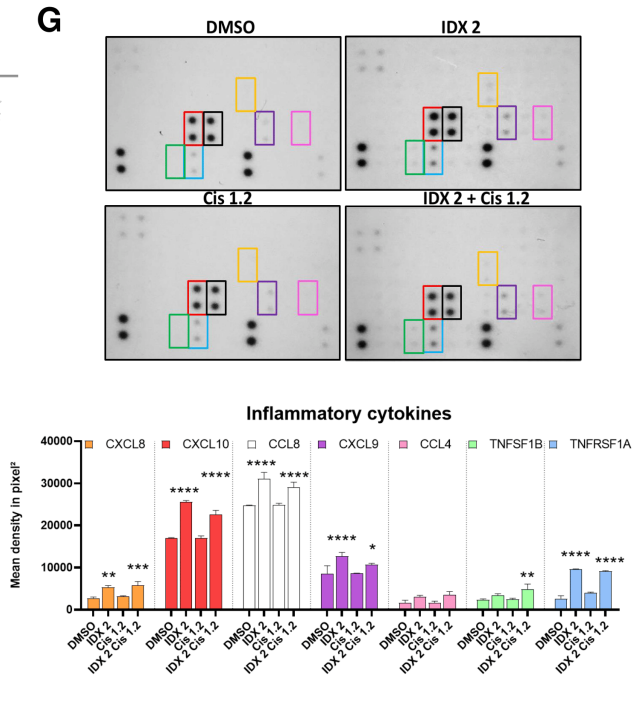
Figure 5
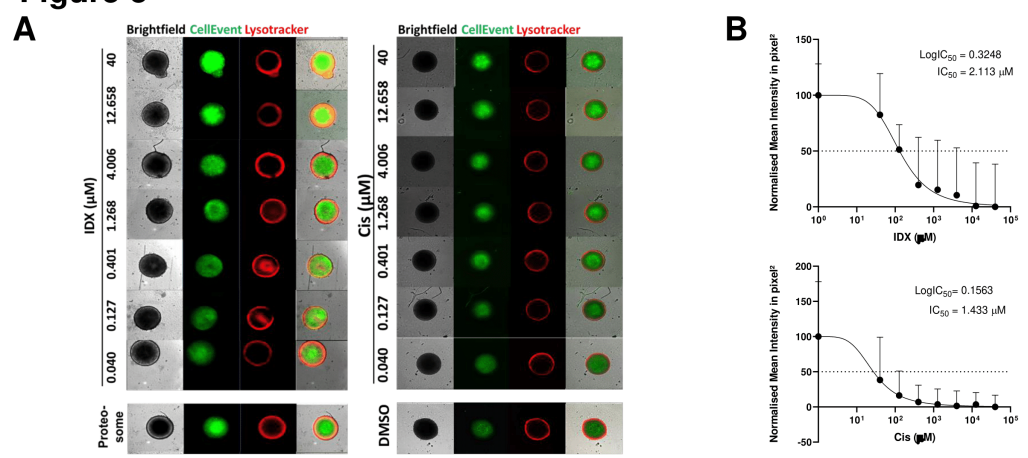

C

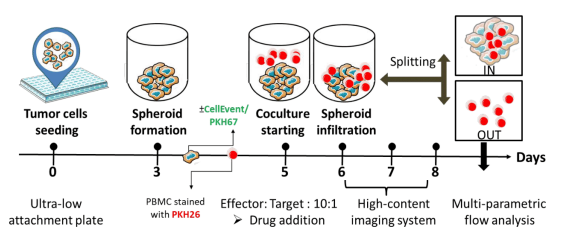

E

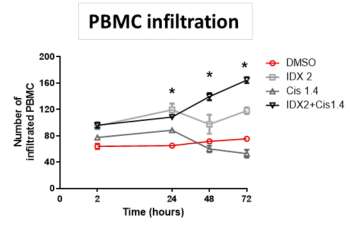

D

F
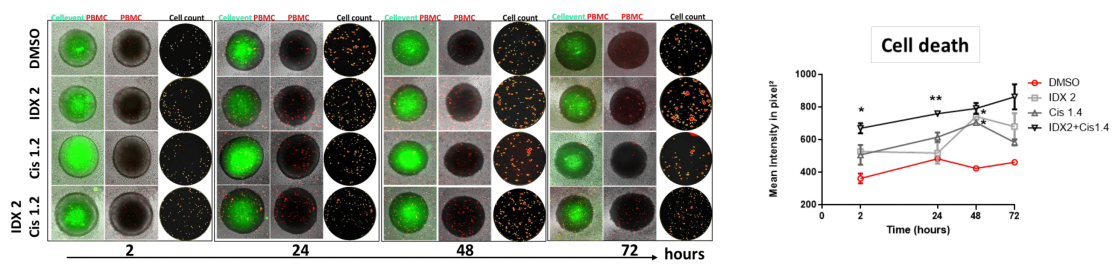

G

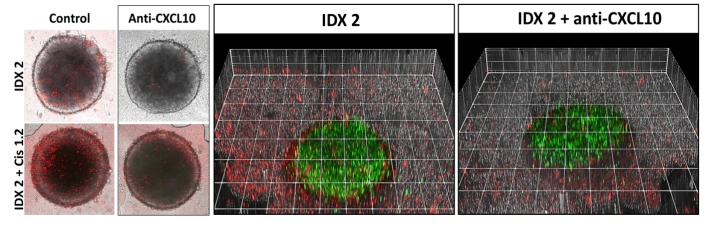

H

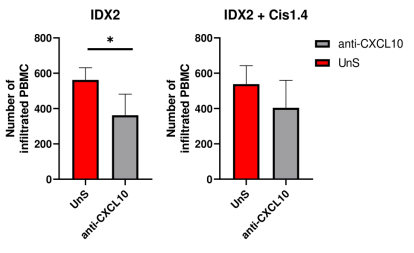


Figure 6

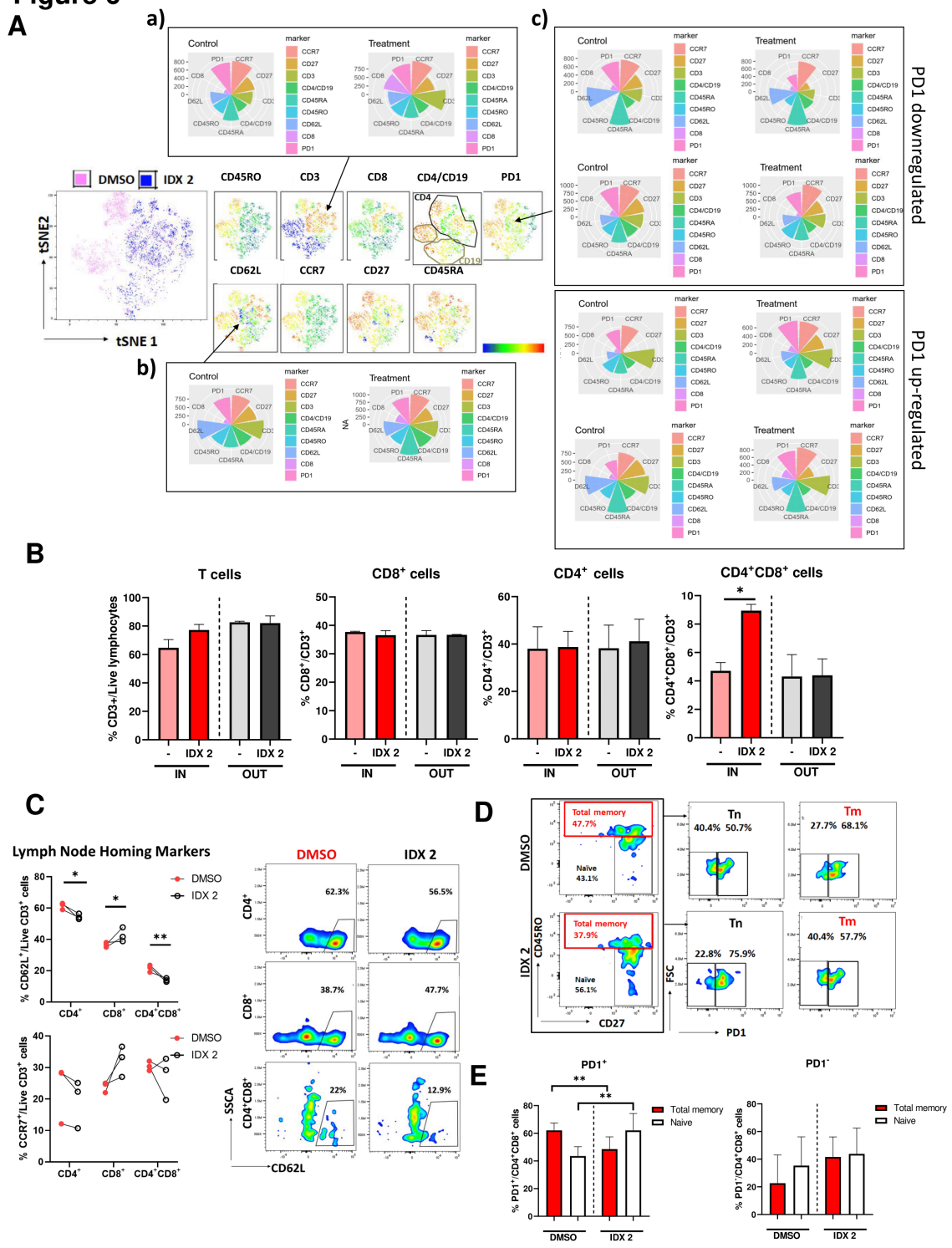


Figure 7

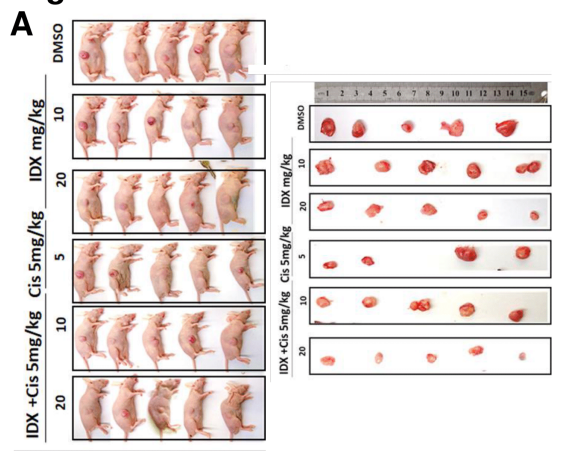

B

C

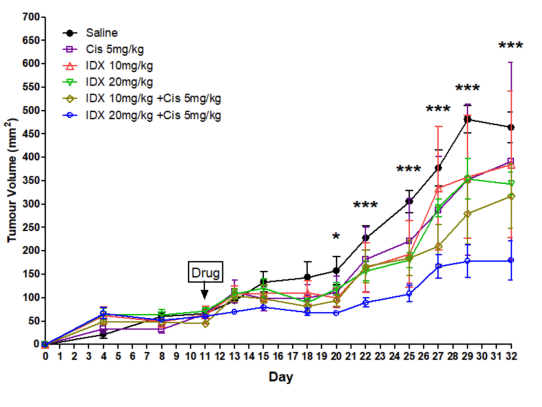

Tumor tissue

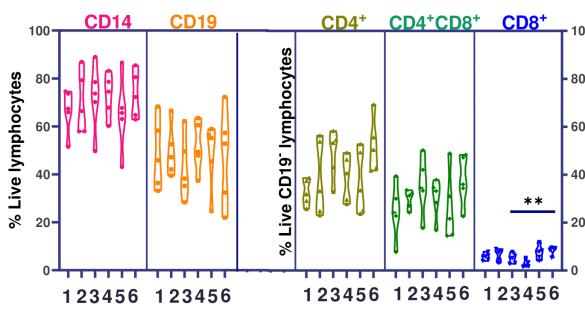

D

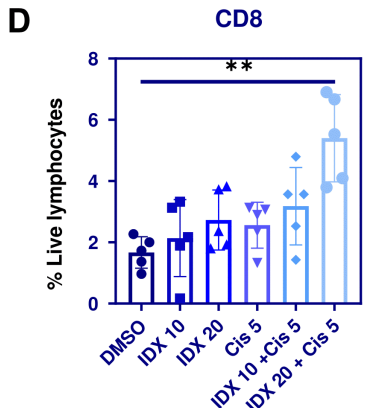

E

G
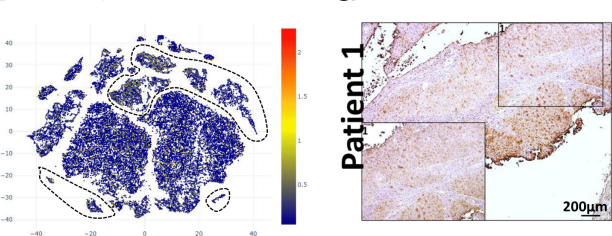

H
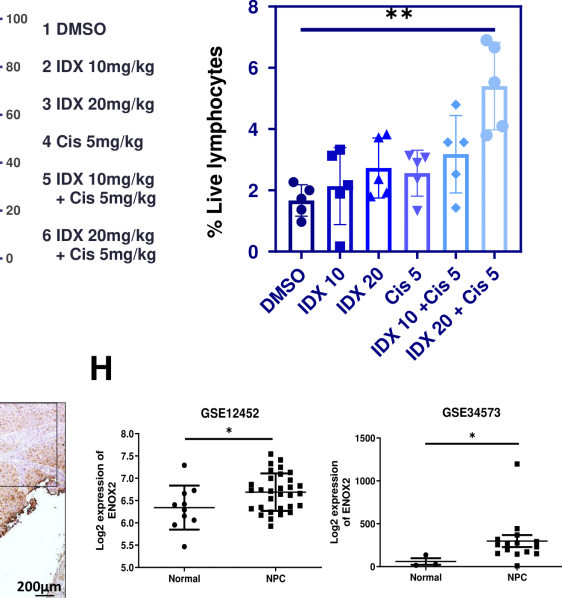

F Expressionof f ENOX2
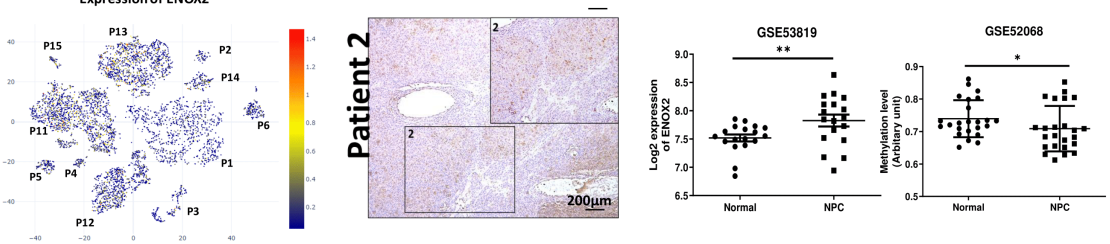